\title{
Pulmonary Embolism in Vaccine-Induced Thrombotic Thrombocytopaenia: Under-Reported?
}

\author{
Authors: \\ ${ }^{*}$ Veerle Leenaerts, ${ }^{1+}$ Michael Staes, ${ }^{1+}$ Jos Callens, ${ }^{2}$ Pieter Monballyu ${ }^{2}$ \\ 1. Faculty of Medicine, Catholic University of Leuven, Belgium \\ 2. Department of Internal Medicine, General Hospital Klina, Brasschaat, Belgium \\ *Correspondence to veerle.leenaerts@student.kuleuven.be \\ +Both first author. \\ Disclosure: The authors have declared no conflicts of interest \\ Received: $\quad 21.07 .21$ \\ Accepted: $\quad 08.09 .21$ \\ Keywords: $\quad$ COVID-19, pulmonary embolism (PE), thrombocytopenia, thrombosis, vaccination. \\ Citation: $\quad$ EMJ Respir. 2021;9[1]:79-85.
}

\begin{abstract}
Vaccine-induced thrombotic thrombocytopaenia (VITT) is a rare, newly described syndrome characterised by thrombocytopaenia and thrombosis 5-24 days after administration of an adenoviral vector-based COVID-19 vaccine. It resembles heparin-induced thrombocytopaenia and, therefore, diagnostics and treatment are similar. Early recognition is essential to avoid potentially fatal outcomes. This article describes a case of VITT with symptomatic cerebral venous-sinus thrombosis and splanchnic vein thrombosis, as well as asymptomatic pulmonary embolism in a 49-year-old male. The authors discuss VITT, focusing on the possibility of pulmonary embolism being under-reported, diagnostic criteria, differential diagnosis, and treatment.
\end{abstract}

\section{INTRODUCTION}

Vaccination is currently the most powerful tool for controlling the COVID-19 pandemic. In large randomised controlled trials, the approved vaccines have shown good efficacy and safety. ${ }^{2}$ However, there is increasing concern about the safety of the adenovirus-based COVID-19 vaccines of AstraZeneca (ChAdOx1 nCoV-19) and Janssen (Ad26.COV2.S). This concern is based on multiple reports of thrombocytopaenia in combination with thrombosis at unusual sites (mainly cerebral venous sinus thrombosis [CVST] and splanchnic vein thrombosis [SVT]) 5-24 days after administration of the adenovirus-based COVID-19 vaccine. Many of these cases were fatal.3,4 This article describes a case of a 49-yearold male with this rare, so-called vaccine-induced

immune thrombotic thrombocytopaenia (VITT), with both symptomatic CVST and SVT and asymptomatic pulmonary embolism (PE), 1 week after receiving the ChAdOx1 nCoV-19 vaccine, with a favourable outcome after treatment. Further, the authors discuss the possibility of under-reporting of PE in VITT.

\section{CASE PRESENTATION}

\section{Initial Presentation}

A 49-year-old white male, with a history of fibromyalgia and nephrolithiasis, presented at the emergency department with fever, vomiting, and abdominal pain radiating to the back. Seven days earlier, he had received his first dose of the ChAdOx1 nCoV-19 vaccine. The day after 
vaccination, he experienced low-grade fever, myalgia, and fatigue for 48 hours. On Day 6 after vaccination, vague pressing lower abdominal pain and vomiting occurred during the night but symptoms had disappeared by the next morning. However, in the evening, abdominal pain recurred and became gradually worse. There were no other complaints. No other family members were ill. He did not take any medications. There were no predisposing factors for thrombosis.

On examination, his temperature was $37.5^{\circ} \mathrm{C}$, blood pressure was $165 / 95 \mathrm{mmHg}$, pulse 70 beats/min, respiratory rate 13 breaths/min, and oxygen saturation was $98 \%$ while the patient was breathing ambient air. He appeared unwell and sweaty, but there was no abdominal tenderness nor other remarkable findings. His weight was $80 \mathrm{~kg}$.
Laboratory results are shown in Table 1. C-reactive protein (CRP) was very high and platelet count was 46.000 cells $/ \mathrm{mm}^{3}$. Severe acute respiratory syndrome coronavirus 2 reverse-transcriptase PCR assay of a nasopharyngeal swab was negative. Urine sediment examination showed no haematuria or pyuria.

An additional CT scan with intravenous contrast of the abdomen was performed, which did not show any abnormalities.

The patient was admitted to the ward and was given broad-spectrum antibiotics, intravenous fluids, and painkillers. Despite the low platelet count, there was no immediate suspicion for VITT.

\section{Inpatient Stay}

On Day 1 after admission, there were no new complaints, but the abdominal pain was still

Table 1: Blood results.

\begin{tabular}{|c|c|c|c|c|c|c|}
\hline $\begin{array}{l}\text { Laboratory } \\
\text { analysis }\end{array}$ & Day 0 & Day 2 & Day 4 & Day 6 & Day 9 & $\begin{array}{l}\text { Reference } \\
\text { value }\end{array}$ \\
\hline $\begin{array}{l}\text { Haemoglobin } \\
(\mathrm{g} / \mathrm{dL})\end{array}$ & 14.9 & 15.1 & 13.0 & 12.9 & 13.8 & $13.5-16.9$ \\
\hline $\begin{array}{l}\text { Platelet count } \\
\left(\times 10^{3} / \mu \mathrm{L}\right)\end{array}$ & 46,000 & 21,000 & 44,000 & 117,000 & 268,000 & $166-308$ \\
\hline $\begin{array}{l}\text { Leukocytes } \\
\left(x 10^{3} / \mu \mathrm{L}\right)\end{array}$ & 7,570 & 4,890 & 11,690 & 8,570 & 7,860 & $3,450-9,760$ \\
\hline $\begin{array}{l}\text { Activated } \\
\text { partial } \\
\text { thromboplastin } \\
\text { time (sec) }\end{array}$ & 37.3 & 44.6 & 34.0 & $\mathrm{~N} / \mathrm{A}$ & $\mathrm{N} / \mathrm{A}$ & $29.0-38.1$ \\
\hline $\begin{array}{l}\text { International } \\
\text { normalised } \\
\text { ratio }\end{array}$ & 1.19 & 1.27 & 1.35 & N/A & $\mathrm{N} / \mathrm{A}$ & $<1.20$ \\
\hline $\begin{array}{l}\text { Fibrinogen } \\
(\mathrm{mg} / \mathrm{dL})\end{array}$ & $\mathrm{N} / \mathrm{A}$ & 3.01 & 1.20 & $\mathrm{~N} / \mathrm{A}$ & $\mathrm{N} / \mathrm{A}$ & $2.00-4.00$ \\
\hline $\begin{array}{l}\text { D-dimer } \\
(\mu \mathrm{g} / \mathrm{mL})\end{array}$ & N/A & $>4.00$ & $>4.00$ & $\mathrm{~N} / \mathrm{A}$ & $\mathrm{N} / \mathrm{A}$ & $<0.50$ \\
\hline AST (U/L) & 31 & 54 & 82 & 82 & 49 & $<40$ \\
\hline $\operatorname{ALT}(U / L)$ & 29 & 41 & 101 & 132 & 116 & $<41$ \\
\hline GGT (U/L) & 52 & 65 & 54 & 100 & 110 & $40-129$ \\
\hline LDH (U/L) & 365 & 331 & 278 & 266 & 250 & $<250$ \\
\hline $\mathrm{CRP}(\mathrm{mg} / \mathrm{L})$ & 184.0 & 131.0 & 29.2 & 12.6 & 7.8 & $<5.0$ \\
\hline cTnT (ng/L) & N/A & N/A & 197 & 284 & 106 & $<14$ \\
\hline
\end{tabular}

ALT: alanine aminotransferase; AST: aspartate aminotransferase; CRP: C-reactive protein; cTnT; cardiac troponin T; GGT: y-glutamyltransferase; LDH: lactate dehydrogenase. 


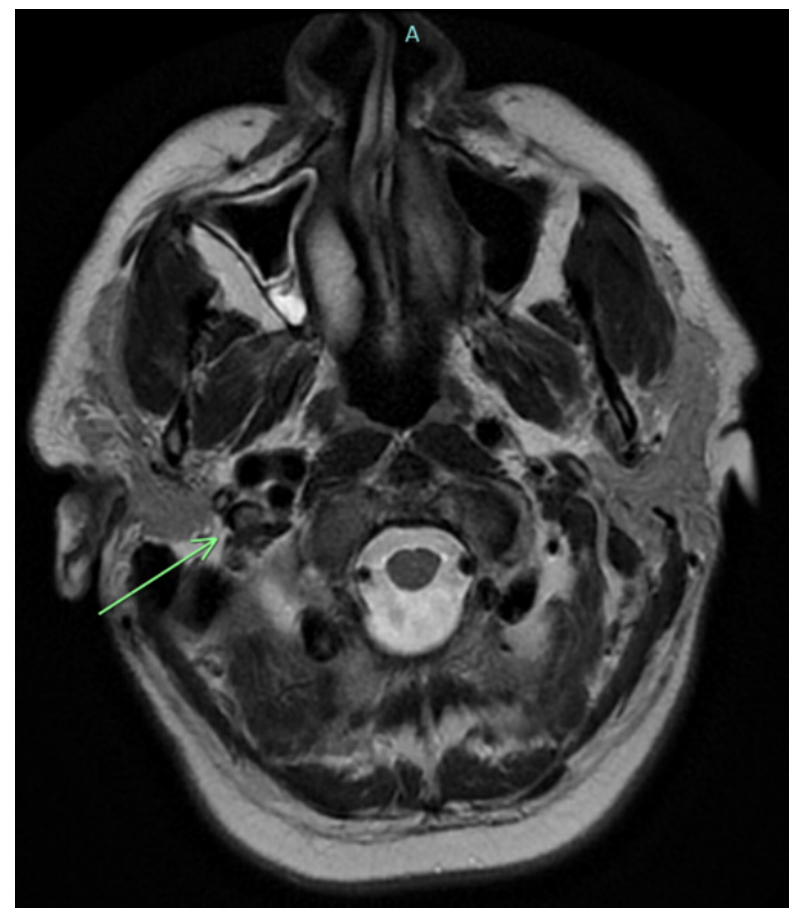

Figure 1: MRI T2-weighted imaging showing a thrombus in the right internal jugular vein.

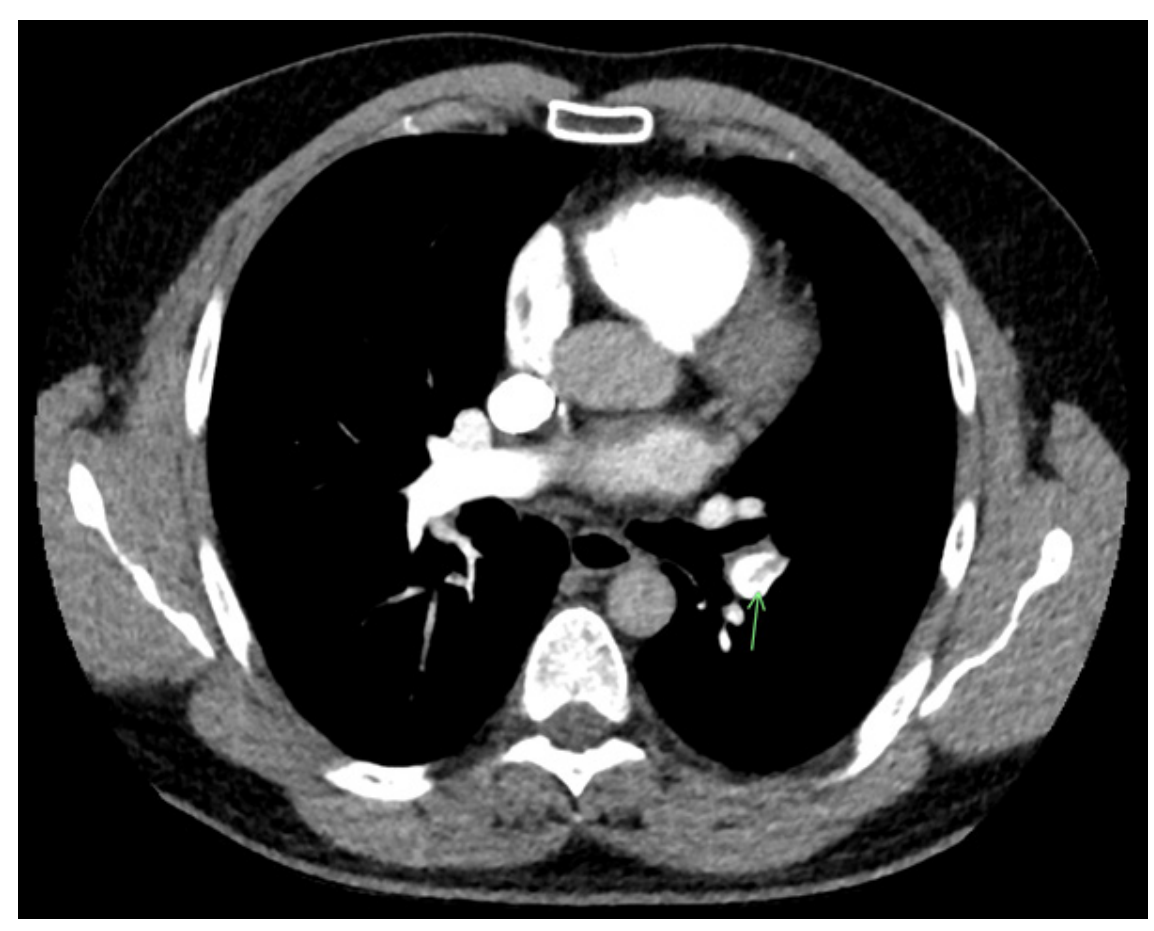

Figure 2: CT pulmonary angiography showing pulmonary embolism in the left lower segmental pulmonary artery.

present. The platelet count had dropped further to 30,000 cells $/ \mathrm{mm}^{3}$, while CRP diminished. There was a slight elevation of the aminotransferases. The coagulation parameters became impaired, with an elevated international normalised ratio, a prolonged activated partial thromboplastin time, and high D-dimer level. The diagnosis of VITT was suspected and the patient was started on a prophylactic dose ( $2.5 \mathrm{mg}$ once daily) of the nonheparin anticoagulant fondaparinux. 
On Day 2 after admission, the patient complained of mild frontal headache. The platelet count decreased to 21,000 cells $/ \mathrm{mm}^{3}$. An urgent magnetic resonance scan of the brain showed a thrombosis of the right sinus transversus and right bulbus jugularis (Figure 1). The patient was transferred to the intensive care unit for further observation and treatment with intravenous Ig (IVIg) at a dose of $1 \mathrm{~g} / \mathrm{kg}$ bodyweight for 2 days, methylprednisolone at a dose of $1 \mathrm{mg} / \mathrm{kg}$ bodyweight for 5 days, and fondaparinux at therapeutic dose ( $7.5 \mathrm{mg}$ once daily).

On Day 3 after admission, mild abdominal pain and headache still existed. Platelet count stabilised, but coagulation abnormalities worsened, with decreased fibrinogen too. A new CT scan of the abdomen was performed, this time with intravenous contrast in the venous phase. It revealed thrombosis of the superior mesenteric vein as well as thrombosis of several intrahepatic veins, causative for the patient's abdominal pain and liver function abnormalities.

On Day 4 after admission, the patient was discharged from intensive care as symptoms diminished and platelet count started to rise. A transthoracic echocardiogram was performed because of an abnormal ECG (new T-wave inversion, inferolateral) taken on admission at the intensive care unit, with mildly elevated troponins. The echocardiogram showed mild pulmonary hypertension, for which a subsequent CT pulmonary angiography was done on Day 6 after admission, which revealed PE in the left lower segmental pulmonary artery (Figure 2). There were no symptoms suggestive for PE.

On Day 7 after admission, the anticoagulant was switched from fondaparinux to the direct oral anticoagulant rivaroxaban. Antibiotics were given for 5 days. On Day 9 after admission, symptoms disappeared completely, platelet count fully recovered, and the patient was discharged from the hospital with rivaroxaban therapy for 3 months.

There was no administration of heparin before or during hospitalisation. On Day 2 after admission, an ELISA for heparin-induced thrombocytopaenia (HIT) was requested. The test turned out to be positive by Day 5 and, therefore, confirmed the authors' diagnosis of VITT.

\section{DISCUSSION}

VITT is a rare, newly described syndrome characterised by the development of antibodies that activate platelet factor 4 (PF4) via the crosslinking of the FcyRIIA receptor, with subsequent thrombosis and thrombocytopaenia 5-24 days after the administration of an adenoviral vector-based COVID-19 vaccine. ${ }^{4}$ It resembles HIT, where antibody formation is induced by heparin exposure. The estimated incidence of VITT is approximately 1 in 150,000 for the ChAdOx1 nCoV-19 vaccine and approximately 1 in 470,000 for the Ad26.COV2.S vaccine, although the incidence rates vary by age group and sex. ${ }^{5}$ VITT is not seen in the mRNAbased COVID-19 vaccines, or other adenoviral vector-based vaccines for COVID-19 (e.g., the Sputnik V [Gam-COVID-Vac]) and Ebola (e.g., the Ad26.ZEBOV-GP, recombinant vaccine). ${ }^{6-9}$

Rapid recognition to prevent a fatal outcome is important. Symptoms vary depending on the site of thrombosis. CVST is the most common site of thrombosis in VITT, followed by SVT (which includes mesenteric, portal, hepatic, and splenic veins) and $P E .3,4,10$ Therefore, the main presenting symptoms are headache, photophobia, abdominal pain, nausea and vomiting, and dyspnoea. ${ }^{11}$ It is well known that patients often have more than one site of thrombosis, although the presence of thrombosis in three different sites, as in the described case, is rare. In two recent case series of 11 and 23 patients, only 2 (18\%) and 1 (4.8\%) had all three (CVST, SVT, and PE, respectively).1,12

In the authors' case, the patient had no symptoms of PE, and the diagnosis was only made in response to a routinely taken ECG showing repolarisation abnormalities. More cases of asymptomatic PE have been reported in VITT. For example, in a case series of 12 patients with VITT with CVST, 3 also had asymptomatic PE. ${ }^{13}$ Garnier et al. ${ }^{14}$ described a case of a 26-yearold female with VITT, with both symptomatic proximal left middle cerebral artery occlusion and asymptomatic SVT and PE after receiving the ChAdOx1 nCoV-19 vaccine. Moreover, the patient used as an index case in Greinacher et al.'s case series had peripheral PE without respiratory symptoms. ${ }^{15}$ Therefore, PE is likely to be underreported in VITT. The clinical relevance of this 
phenomenon is still unknown. In patients with proven VITT and thrombosis in other sites, there seem to be no direct therapeutic consequences, but follow-up may be different since PE can have long-term complications.

VITT should be suspected in patients developing signs or symptoms of thrombocytopaenia or thrombosis during an appropriate time-frame following the COVID-19 vaccination. According to the American Society of Hematology (ASH), five criteria must be met to make a definitive diagnosis of VITT:

1. administration of a COVID-19 vaccine 4-42 days prior to symptom onset;

2. any venous or arterial thrombosis;

3. thrombocytopaenia (platelet count $\left.<150 \times 10^{9} / \mathrm{L}\right)$;

4. markedly elevated D-dimer (>4 times upper limit of normal); and

\section{5. positive PF4 ELISA. ${ }^{16}$}

Although a positive PF4 ELISA is necessary to confirm diagnosis, no single ELISA method detects all VITT cases. In a large prospective study, $2.7 \%$ of patients meeting all other diagnostic VITT criteria had a negative PF4 ELISA result. These cases were considered 'probable VITT' and received regular VITT therapy. ${ }^{17} \mathrm{~A}$ second ELISA should be considered if there is a strong clinical suspicion of VITT. ${ }^{18}$ Other types of PF4 antibody tests, such as the serotonin release assay or rapid HIT assays, are not reliable enough to exclude or confirm the diagnose of VITT.12 To reduce the chance of false negative results, it is important to collect all blood samples for VITT testing before the administration of any treatment (especially IVIg). ${ }^{6}$

In patients with suspected VITT, alternative diagnoses should be considered too. Table 2 provides a non-exhaustive list of differential diagnoses. 6,17 VITT belongs to a spectrum of platelet-activating anti-PF4 disorders. This spectrum also includes HIT, in which the production of PF4 antibodies follows heparin exposure. A clinical history is usually sufficient to distinguish between VITT and HIT. An even rarer condition is spontaneous HIT, where there is no known preceding heparin exposure. VITT could be seen as a form of spontaneous HIT triggered by vaccination. The combination of thrombocytopaenia and thrombosis is also seen in haemolytic uraemic syndrome, thrombotic thrombocytopaenic purpura, disseminated intravascular coagulation, paroxysmal nocturnal haematuria, and haematologic malignancies. ${ }^{6}$ Moreover, a COVID-19 infection itself carries a high risk of thrombosis, often in combination with thrombocytopaenia. ${ }^{19}$ Thrombocytopaenia without thrombosis is seen in a lot of diseases. Vaccine-associated immune thrombocytopaenic purpura is mainly seen after vaccination for measles, mumps, and rubella, but is also described after vaccination for COVID-19. ${ }^{20-23}$ A lot of infections can induce thrombocytopaenia, but VITT cannot be ruled out based on the presence of highly elevated CRP. As in the authors' case, VITT is sometimes associated with high levels of CRP., ${ }^{1,24}$

The treatment of VITT is similar to that of HIT and is based on high-dose IVIg and anticoagulation. The therapy should be initiated when there is high clinical suspicion for VITT (confirmed thrombosis and thrombocytopaenia after recent vaccination) before the results of the anti-PF4 antibody testing is known. Urgent administration of IVIg in a dose of $1 \mathrm{mg} / \mathrm{kg}$ of ideal bodyweight for 2 days is recommended. Although there is no evidence, the use of heparin may be harmful and, therefore, non-heparin anticoagulants are preferred. The choice of anticoagulant is based on preferred route of administration, availability, duration of action (no long-acting anticoagulants when high bleeding risk or there is need for invasive procedures), availability of antidotes (especially with a high bleeding risk), price, and reimbursement criteria. Options are direct parenteral (argatroban, bivalirudin) or oral (dabigatran) thrombin inhibitors, direct oral anti-Xa inhibitors (edoxaban, apixaban, rivaroxaban), low-molecular-weight heparinoid devoid of heparin (danaparoid), and selective factor-Xa inhibitor (fondaparinux). The authors' patient was started on fondaparinux, but after confirmation of the diagnosis of PE, they could switch to rivaroxaban because it is reimbursed for this indication in Belgium. Both the British Society of Haematology (BSH) and $\mathrm{ASH}$ recommend continuing systemic anticoagulation for a minimum of 3 months in patients with documented thrombosis. ${ }^{6}$ 
Table 2: Differential diagnosis of vaccine-induced thrombotic thrombocytopaenia.

\begin{tabular}{|c|c|}
\hline Thrombosis & Thrombocytopaenia \\
\hline - COVID-19 infection & - Drug-/toxin-induced thrombocytopaenia \\
\hline - Hereditary thrombophilia & $\begin{array}{l}\text { - Thrombocytopaenia reactive to an acute medical } \\
\text { condition (sepsis, viral infection, etc.) }\end{array}$ \\
\hline - Acquired thrombophilia & • ITP, inclusive post-vaccine ITP \\
\hline - Antiphospholipid syndrome & $\begin{array}{l}\text { - Hypersplenism, e.g., secondary to portal hypertension in } \\
\text { liver cirrhosis }\end{array}$ \\
\hline - Chronic DIC & \multirow[t]{2}{*}{ - Vitamin deficiencies } \\
\hline $\begin{array}{l}\text { - Idiopathic with possible risk factors, such as malignancy, } \\
\text { smoking, female gender, oestrogen therapy or pregnancy, } \\
\text { immobilisation }\end{array}$ & \\
\hline \multicolumn{2}{|l|}{ Thrombosis and thrombocytopaenia } \\
\hline \multicolumn{2}{|l|}{ - COVID-19 infection } \\
\hline \multicolumn{2}{|l|}{ • HIT } \\
\hline \multicolumn{2}{|l|}{$\cdot$ TTP } \\
\hline \multicolumn{2}{|l|}{ - HUS and aHUS } \\
\hline \multicolumn{2}{|l|}{ - $\mathrm{PNH}$} \\
\hline - Malignant haematologic disorders & \\
\hline
\end{tabular}

aHUS: atypical haemolytic uraemic syndrome; DIC: disseminated intravascular coagulation; HIT: heparin-induced thrombocytopaenia; HUS: haemolytic uraemic syndrome; ITP: immune thrombocytopaenic purpura; PNH: paroxysmal nocturnal haematuria; TTP: thrombotic thrombocytopaenic purpura.

The described patient was also given corticosteroids, which is not recommended in the current guidelines. Platelet transfusions should be avoided as they can worsen thrombus formation. They should be only considered when there is lifethreatening bleeding. ${ }^{5,6}$ Further, close monitoring and supportive therapy (painkillers when there is pain, oxygen when there is hypoxia, antiemetics when vomiting, thrombectomy in exceptional circumstances, etc.) are indicated. Finally, adenoviral vector-based COVID-19 vaccines should be avoided in patients with confirmed VITT, but mRNA-based COVID-19 vaccines are considered safe as they are not associated with VITT. ${ }^{6}$ The ideal interval between the diagnosis of VITT and administration of an mRNA-based COVID-19 vaccine is not known. ${ }^{5}$

\section{CONCLUSION}

VITT is a rare but potentially fatal complication of adenoviral vector-based COVID-19 vaccines. CVST is the most common site of thrombosis in VITT, followed by SVT and PE. The authors reported a case of VITT in a 49-year-old male, with both symptomatic CVST and SVT and also asymptomatic PE. Based on existing literature, asymptomatic PE is not uncommon in VITT. Therefore, PE seems to be under-reported in VITT. The clinical relevance of this phenomenon is yet undetermined, but clinical awareness is needed. The authors suggest performing a CT scan of the chest with pulmonary angiography in every patient with a proven VITT to rule out PE.

\section{References}

1. Schultz NH et al. Thrombosis and thrombocytopenia after ChAdOx1 $\mathrm{nCoV}$-19 vaccination. $\mathrm{N}$ Engl J Med. 2021;384(22):2124-30.
2. Long B et al. Thrombosis with thrombocytopenia syndrome associated with COVID-19 vaccines. Am J Emerg Med. 2021;49:58-61.
3. Cines DB, Bussel JB. SARS-CoV-2 vaccine-induced immune thrombotic thrombocytopenia. N Engl J Med. 2021;384(23):2254-56. 
4. Franchini $M$ et al. COVID-19 vaccineassociated immune thrombosis and thrombocytopenia (VITT): diagnosis and therapeutic recommendations for a new syndrome. Eur J Haematol. 2021;107(2):173-80.

5. Siegler JE et al. Cerebral vein thrombosis with vaccineinduced immune thrombotic thrombocytopenia. Stroke. 2021;52(9):3045-53.

6. Aleem A, Nadeem AJ. Coronavirus (COVID-19) vaccine-induced immune thrombotic thrombocytopenia (VITT). 2021. Available at: https://www.ncbi. nlm.nih.gov/books/NBK570605/. Last accessed: 28 August 2021.

7. Cazzola M et al. Controversy surrounding the Sputnik V vaccine. Respir Med. 2021;187:106569.

8. Zhu FC et al. Safety and immunogenicity of a recombinant adenovirus type- 5 vector-based Ebola vaccine in healthy adults in Sierra Leone: a single-centre, randomised, double-blind, placebocontrolled, phase 2 trial. Lancet. 2017:389(10069):621-8.

9. Matz KM et al. Ebola vaccine trials: progress in vaccine safety and immunogenicity. Expert Rev Vaccines. 2019;18(12):1229-42.

10. Gupta A et al. COVID-19 vaccineinduced thrombosis and thrombocytopenia- a commentary on an important and practical clinical dilemma. Prog Cardiovasc Dis. 2021;67:105-7.

11. Arepally GM, Ortel TM. Vaccineinduced immune thrombotic thrombocytopenia (VITT): what we know and do not know. Blood. 2021;138(4):293-8

12. Scully $M$ et al. Pathologic antibodies to platelet factor 4 after $\mathrm{ChAdOx} 1$ nCoV-19 vaccination. N Engl J Med. 2021;384(23):2202-11.

13. See I et al. US case reports of cerebra venous sinus thrombosis with thrombocytopenia after Ad26.CoV2.S vaccination, March 2 to April 21, 2021. JAMA. 2021;325(24):2448-56.

14. Garnier M et al. Imaging of Oxford/ AstraZeneca ${ }^{\circledast}$ COVID-19 vaccineinduced immune thrombotic thrombocytopenia. Diagn Interv Imaging. 2021;DOI:10.1016/j. diii.2021.04.005

15. Greinacher A et al. Thrombotic thrombocytopenia after $\mathrm{ChAdOx} 1$ nCov-19 vaccination. N Engl J Med. 2021;384(22):2092-2101.

16. Bussel JB et al. Thrombosis with thrombocytopenia syndrome (also termed vaccine-induced thrombotic thrombocytopenia). 2021. Available at: https://www.hematology.org/ covid-19/vaccine-induced-immunethrombotic-thrombocytopenia. Last accessed: 25 August 2021.
17. Pavord $\mathrm{S}$ et al. Clinical features of vaccine-induced immune thrombocytopenia and thrombosis. N Engl J Med. 2021:DOI:10.1056/ NEJMoa2109908.

18. Platton $\mathrm{S}$ et al. Evaluation of laboratory assays for anti-platelet factor 4 antibodies after ChAdOx1 nCOV-19 vaccination. J Thromb Haemost. 2021;19(8):2007-13.

19. Gómez-Mesa JE et al. Thrombosis and coagulopathy in COVID-19. Curr Probl Cardiol. 2021;46(3):100742.

20. O'Leary ST et al. The risk of immune thrombocytopenic purpura after vaccination in children and adolescents. Pediatrics. 2012;129(2):248-55.

21. Tarawneh $\mathrm{O}$, Tarawneh $\mathrm{H}$. Immune thrombocytopenia in a 22-yearold post COVID-19 vaccine. Am J Hematol. 2021;96(5):E133-4.

22. Shah SRA et al. COVID-19 vaccination associated severe immune thrombocytopenia. Exp Hemato Oncol. 2021;10(1):42

23. Koch $\mathrm{M}$ et al. Secondary immune thrombocytopenia (ITP) associated with ChAdOx1 COVID-19 vaccination - a case report. TH Open. 2021;5(3):e315-8.

24. Beelen $\mathrm{K}$ et al. Trombose en trombopenie na COVID-19vaccinatie. Ned Tijdschr Geneeskd. 2021;165:D6055. (In Dutch) 